\title{
Osteosíntesis con placa en fracturas de Rolando
}

\author{
O. Sáez de Ugarte Sobrón ${ }^{(1)}$, A. Cruchaga Celada ${ }^{(1)}$, \\ I. Astobiza Bretones ${ }^{(2)}$, I. Gutiérrez SÁNCheZ ${ }^{(1)}$, I. Herreros Ugarte ${ }^{(2)}$, \\ F. LABAYRU ETXEBARRía ${ }^{(1)}$
}

Hospital de Galdakao. Servicio de Traumatología

(1) Adjunto de TRAumatología

(2) MÉdico Residente de TRAumatología

\author{
Correspondencia: \\ Dr. Oskar Sáez de Ugarte Sobrón \\ Hospital de Galdakao. Servicio de Traumatología \\ Barrio Labeaga, s/n \\ Galdakao, Vizcaya, España \\ Teléf: 944007009 \\ FAX: 944007132 \\ email: oskarsmooky@yahoo.es
}

Objetivo: El objetivo del trabajo es valorar los resultados de las fracturas de Rolando tratadas mediante reducción y osteosíntesis.

Material y Método: Se presentan 7 casos de fracturas de Rolando (3 conminutas y 4 de trazo en «Y») tratadas mediante reducción abierta y osteosíntesis con placa.

Resultados: Los resultados tras una media de seguimiento de 3 años muestran una ausencia de artrosis radiológica, sin clínica dolorosa.

Conclusión: Creemos que, a pesar de la dificultad técnica que representan, sobre todo los casos más conminutos, el tratamiento mediante cirugía abierta de estas lesiones ofrece al cirujano la posibilidad de un buen control de la reducción articular y una buena estabilidad primaria de la fractura para permitir una rehabilitación precoz.

Palabras clave: Rolando, primer metacarpiano. fracturas de metacarpianos.
Objetive: The background of this work was to value the results of the Rolando's fractures treated by open reduction and internal fixation.

Material and Method: Seven cases of Rolando's fracture are described (3 comminuted and 4 «Y-shaped»), all treated by open reduction and plate osteosynthesis.

Results: The results show an absence of radiological arthritis and no painful clinical symptoms, after a mean of three years follow-up.

Conclusions: We believe that, despite the technical difficulty that this condition presents, especially in the most comminuted cases, treating these injuries via open surgery helps the surgeon to control the articular reduction and provides a good level of primary stability for the fracture, leading to early rehabilitation.

Key Words: Rolando's fracture, first metacarpal, metacarpal fractures.

\section{INTRODUCCIÓN}

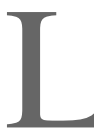

as fracturas de Rolando fueron descritas originalmente en 1910 por Silvio Rolando como fracturas articulares de la base del primer metacarpiano con morfología en
«Y», observadas en obreros del puerto de Genova ${ }^{1}$. Sin embargo, hoy día, en general, se incluyen en este grupo todas aquellas fracturas intraarticulares de la base del primer metacarpiano que asocian mayor o menor grado de conminución, y/o escalones articulares significati- 


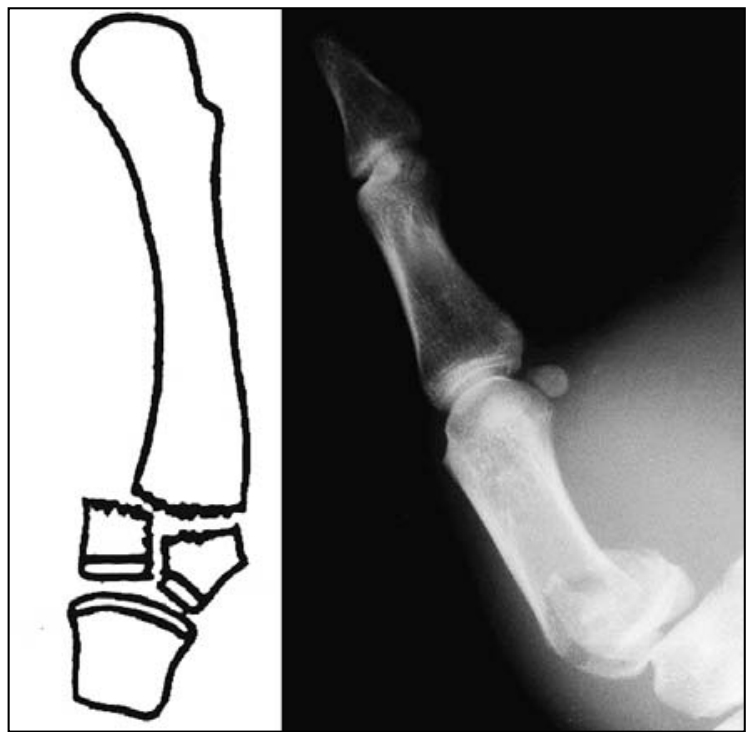

Figura 1. Fractura de Rolando con trazo en «Y».

$\operatorname{vos}^{2}$. Todo esto implica que el tratamiento de estas lesiones supone un reto para el cirujano (Figuras 1 y 2).

No existe consenso acerca de cuál es el tratamiento más adecuado para estas fracturas. A lo largo del tiempo, se han utilizado varios métodos, que van desde la reducción cerrada e inmovilización con yeso hasta la osteosíntesis con placa, pasando por la fijación percutánea con agujas, o los fijadores externos ${ }^{3,4}$. En nuestro servicio, habiendo usado con anterioridad los otros métodos (menos la fijación externa), actualmente optamos en buena parte de los casos por la reducción abierta y fijación con placa, con el fin de, por un lado, intentar lograr una reducción anatómica de la articulación, y por otro, posibilitar la rehabilitación precoz de la mano lesionada (Figura 3), de acuerdo con los criterios actuales generales del tratamiento de una fractura articular.

\section{MATERIAL Y MÉTODO (Tabla I)}

Se han revisado retrospectivamente 7 casos de fracturas de Rolando, tratadas en el Servicio mediante reducción abierta y osteosíntesis con placa, entre los años 2002 y 2005 . Tres casos eran de tipo conminuto, y cuatro de trazo en $« Y »$.

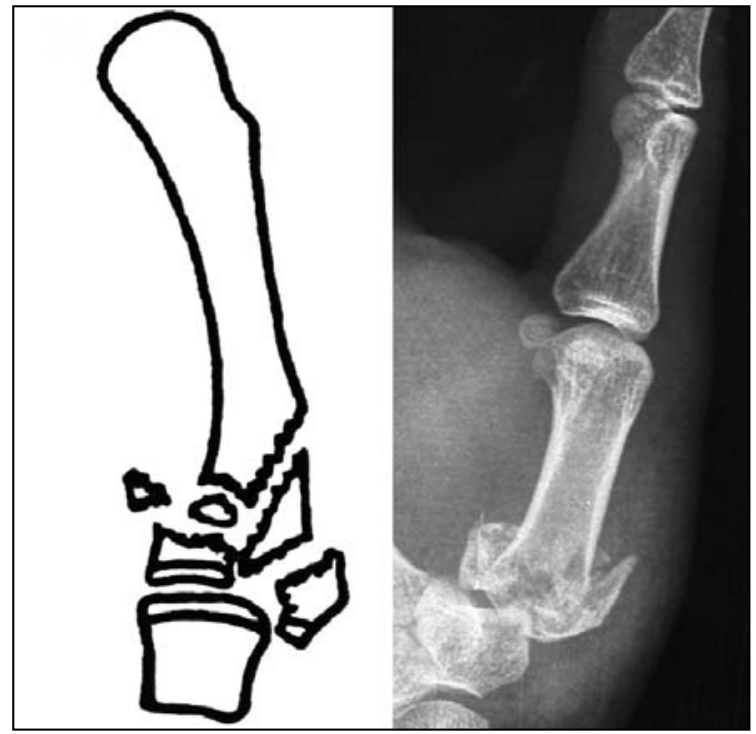

Figura 2. Fractura de Rolando conminuta.

La edad media de los pacientes fue de 36 años (rango 21-66). En 6 de los 7 se indicó la cirugía como tratamiento inicial de la fractura, y en 1 caso como solución tras un desplazamiento secundario que tuvo lugar tras 10 días de inmovilización con yeso. En todos los pacientes se realizó un abordaje quirúrgico palmar en «J» a nivel del margen radial de la eminencia tenar que permitió el control de la reducción articular, empleando para la osteosíntesis una placa de minifragmentos de titanio de bajo perfil. No fue necesario el empleo de injerto óseo en ningún caso. Dada la conminución que presentan en ocasiones estas fracturas, muchas veces en pacientes osteoporóticos, creemos que la nueva generación de placas de osteosíntesis, con tor-

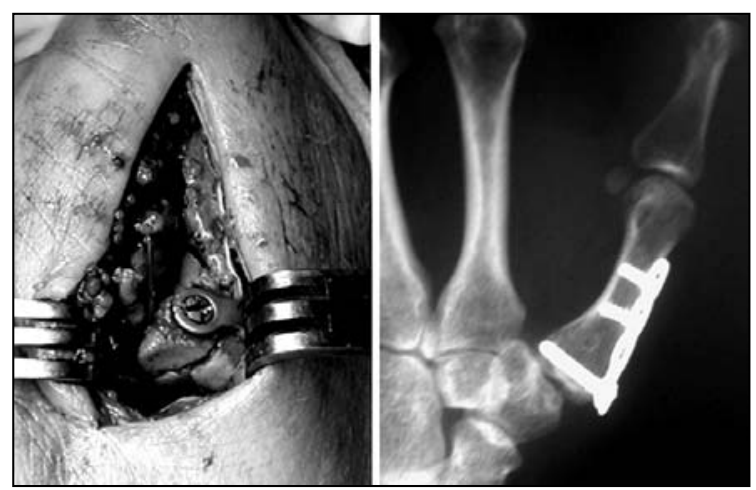

Figura 3. Reducción anatómica de una fractura conminuta de Rolando, y resultado radiológico a los 4 años, sin signos artrósicos. 


\section{Tabla I - SERIE DE CASOS DE FRACtURAS DE ROLANDO tRATADAS MEDIANTE REDUCCIÓN ABIERTA Y OSTEOSÍNTESIS CON PLACA}

\begin{tabular}{|c|c|c|c|c|c|c|c|c|c|}
\hline $\begin{array}{c}\mathbf{N}^{\circ} \\
\text { caso }\end{array}$ & $\begin{array}{l}\text { Tipo de } \\
\text { fractura }\end{array}$ & $\begin{array}{l}\text { Edad } \\
\text { (años) }\end{array}$ & $\begin{array}{c}\text { Escalones } \\
\text { articulares } \\
\text { iniciales } \\
\text { postquirúrgicos }\end{array}$ & $\begin{array}{c}\text { Inmovilización } \\
\text { postoperatoria } \\
\text { (días) }\end{array}$ & \begin{tabular}{|c|} 
Tiempo de \\
seguimiento \\
(meses)
\end{tabular} & $\begin{array}{c}\text { Dolor al } \\
\text { final del } \\
\text { seguimiento }\end{array}$ & $\begin{array}{l}\text { Movilidad al } \\
\text { final del } \\
\text { seguimiento }\end{array}$ & \begin{tabular}{|c|} 
Resultado \\
radiológico al final \\
del seguimiento \\
(¿Rizartrosis?)
\end{tabular} & Comentarios \\
\hline 1 & Conminuta & 24 & $2 \mathrm{~mm}$ & 7 & 20 & $\begin{array}{l}\text { A grandes } \\
\text { esfuerzos }\end{array}$ & Completa & $\begin{array}{l}\text { NO rizartrosis. } \\
\text { Tornillo } \\
\text { proximal largo }\end{array}$ & - \\
\hline 2 & $E n$ « Y » & 24 & $0 \mathrm{~mm}$ & 0 & 19 & NO & $\begin{array}{c}\text { Pérdida leve } \\
\text { de flexo- } \\
\text { aducción }\end{array}$ & NO rizartrosis & - \\
\hline 3 & $E n$ « Y » & 21 & $0 \mathrm{~mm}$ & 17 & 63 & NO & Completa & NO rizartrosis & $\begin{array}{c}\text { Sección } \\
\text { tendinosa del } \\
\text { flexor carpi } \\
\text { ulnaris }\end{array}$ \\
\hline 4 & $E n$ « Y » & 35 & $0 \mathrm{~mm}$ & 0 & 24 & NO & Completa & NO rizartrosis & - \\
\hline 5 & Conminuta & 44 & $0-1 \mathrm{~mm}$ & 5 & 32 & NO & $\begin{array}{c}\text { Pérdida leve } \\
\text { de flexo- } \\
\text { aducción }\end{array}$ & NO rizartrosis & - \\
\hline 6 & $E n$ EY & 66 & $0 \mathrm{~mm}$ & 28 & 32 & NO & Completa & $\begin{array}{l}\text { NO rizartrosis. } \\
\text { Tornillo } \\
\text { proximal largo }\end{array}$ & $\begin{array}{c}\text { Estabilidad } \\
\text { primaria } \\
\text { postquirúrgica } \\
\text { precaria }\end{array}$ \\
\hline 7 & Conminuta & 51 & $1 \mathrm{~mm}$ & 13 & 48 & NO & Completa & NO rizartrosis & - \\
\hline
\end{tabular}

nillos roscados a placa, es una opción muy a tener en cuenta.

El tiempo medio de inmovilización postoperatoria fue de 10 días (rango 0-28). En 6 de los 7 pacientes se consiguió una buena estabilidad primaria, como para permitir el inicio inmedia-

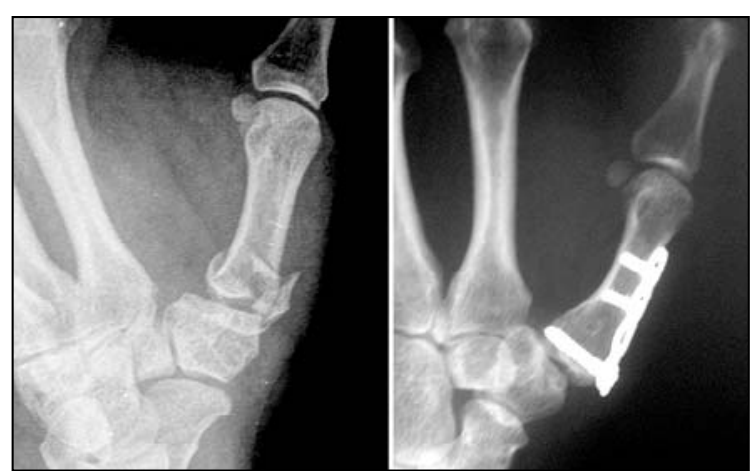

Figura 4. Fractura conminuta de Rolando tratada mediante síntesis con placa. A los 4 años de la intervención quirúrgica, la paciente se encuentra asintomática y no existen signos radiológicos de rizartrosis. to de la rehabilitación, incidiendo de manera especial en ejercicios para trabajar la posición de pinza, tanto la de oposición como la lateral, aunque en uno de éstos se mantuvo una férula de yeso porque presentaba lesiones asociadas de la musculatura extrínseca flexora, y en 1 caso se mantuvo una inmovilización con yeso durante 4 semanas por no conseguir una osteosíntesis suficientemente estable.

El tiempo medio de seguimiento ha sido de 34 meses (rango 19-63).

\section{RESULTADOS}

Se han analizado los resultados tanto desde un punto de vista clínico como radiológico (Figura 4).

Clínicamente sólo un paciente refiere molestias leves al realizar esfuerzos, y no se ha apreciado disminución significativa en los diferen- 
Tabla II - MOVILIDAD DEL PULGAR EN LA ÚLTIMA REVISIÓN REALIZADA, COMPARADA CON LA MANO SANA CONTRALATERAL

\begin{tabular}{|c|c|c|c|c|c|c|}
\hline \multirow{2}{*}{$\mathbf{N}^{\circ}$ Caso } & \multicolumn{3}{|c|}{$\begin{array}{c}\text { Movilidad en la mano lesionada } \\
\text { (última revisión) }\end{array}$} & \multicolumn{3}{c|}{ Movilidad en la mano contralateral } \\
(sana)
\end{tabular}

tes arcos de movimiento (Tabla II), tomando como referencia la mano sana contralateral: en dos casos existía una pérdida de $2-3 \mathrm{~mm}$ en la flexo-aducción del pulgar (se considera que la flexo-aducción del pulgar es normal cuando el pulpejo del pulgar puede contactar con la articulación metacarpofalángica del $5^{\circ}$ dedo, es decir, cuando la distancia entre ambos puntos es de 0 milímetros). Ningún paciente presentaba pérdida de apertura de la primera comisura.

En lo concerniente al resultado radiológico, no se han detectado signos de rizartrosis en ningún caso, si bien el tiempo medio de seguimiento tampoco es excesivo. No se visualizaban escalones articulares en 5 pacientes, un caso presentaba un escalón de $1 \mathrm{~mm}$ y otro caso de $2 \mathrm{~mm}$.

No hay ningún caso de infección de la osteosíntesis ni de pérdida de reducción de la fractura con el paso del tiempo. En 2 casos los tornillos proximales de la placa eran algo más largos de lo normal, pero no han causado dolor a los pacientes, y no ha sido necesario retirarlos.

\section{DISCUSIÓN}

Al hacer una revisión bibliográfica del tema, no se han encontrado muchas publicacio- nes que traten específicamente, o por separado, sobre el tratamiento de las fracturas de Rolando ${ }^{2,4,7,8}$; a menudo, en el análisis de resultados, las fracturas de Rolando aparecen mezcladas con fracturas de Bennett, ó incluso con fracturas extraarticulares del primer metacarpiano $^{3,5,6,9,10}$.

En el estudio de van Niekerk y Ouwens ${ }^{5}$ revisaron 23 fracturas de la base del primer metacarpiano, 7 de las cuales eran fracturas de Rolando. Realizaron fijación percutánea con agujas, no logrando una reducción adecuada articular en 4 de los 7 casos. En la revisión a los 6 años, 4 pacientes presentaban artrosis grave de la trapeziometacarpiana, y 3 de las 7 fracturas de Rolando operados tenían dolor. Estos autores finalizan su artículo recomendando la reducción anatómica de la fractura en la medida de lo posible.

En el trabajo de Langhoff et al. ${ }^{6}$ sobre 17 fracturas de Rolando, 14 presentaban desplazamiento interfragmentario, y los autores optaron por realizar reducción abierta en 11 casos. Sin embargo, la osteosíntesis fue llevada a cabo con agujas de Kirschner, que no suelen proporcionar una buena estabilidad primaria. Consiguieron un buen control inicial de la reducción articular en 9 casos, y, aunque no pudieron encontrar una relación estadísticamente significa- 
tiva entre la calidad de la reducción inicial y clínica dolorosa tras cinco años de seguimiento, concluyen que se debe intentar la reducción anatómica de la fractura.

Surzur et al. ${ }^{8}$ publicaron una revisión sobre 138 fracturas de la base del primer metacarpiano. Los 16 casos de fractura de Rolando de la serie fueron tratados así: 10 con reducción cerrada y agujas percutáneas, 3 con fijador externo, y 3 mediante reducción abierta y fijación con placa. Si analizamos juntas todas las fracturas de Rolando (trazo clásico en «Y» y las conminutas), vemos que a los 7 años de seguimiento, 9 de los 16 (el 56\%) presentaban artrosis moderada o grave, y 7 de los 16 (el 44\%) un resultado funcional «no bueno», según los parámetros empleados por los autores para la medición de resultados. Concluyen que debe intentarse una reducción abierta de la articulación y una osteosíntesis directa si el tamaño de los fragmentos lo permite.

Finalmente Bruske et al. ${ }^{10}$ estudiaron un grupo de 35 fracturas de la base del primer metacarpiano, 14 de las cuales correspondían a fracturas de Rolando. Llevaron a cabo un estudio comparativo entre tratamiento percutáneo con agujas y reducción abierta más fijación con placa, pero a la hora de analizar los resultados no distinguen entre fracturas de Bennett y de Rolando, sino que se limitan a comparar los dos tipos de osteosíntesis independientemente del tipo de fractura. No encuentran diferencias destacables entre ambos métodos, salvo que los casos tratados con reducción abierta y osteosíntesis más fijación con placa presentaban una mejor flexoaducción del pulgar que los tratados con agujas.

\section{CONCLUSIONES}

El abordaje abierto de estas fracturas nos permite un buen control de la reducción articular, y la osteosíntesis con placa de titanio de bajo perfil ofrece, en general, una estabilidad suficiente para permitir el inicio inmediato de la rehabilitación.

Creemos que este método de tratamiento ofrece buenos resultados clínicos y radiológicos, con pocas complicaciones asociadas, aunque puede resultar complejo de llevar a cabo, especialmente en las fracturas de Rolando con importante conminución.

\section{BIBLIOGRAFÍA}

1. Rolando S. Traslated by Meals $\mathrm{R}$. The classic : Fracture of the base of the first metacarpal and a variation that has not yet been described. Clin Orthop, 2006; 445: 15-8.

2. Soyer AD. Fractures of the base of the first metacarpal: current treatment options. J Am Acad Orthop Surg, 1999; 7: 403-12.

3. Demir E, Unglaub F, Wittemann M. Osteosynthetisch versorgte intraartikuläre Fracturen der Metakarpale-I-Basis. Unfallchirurg, 2006; 109: 13-21.

4. Proubasta I. Rolando's frac- ture of the first metacarpal. Treatment by external fixation. J Bone Joint Surg B, 1992; 74: 416-7.

5. van Niekerk JL, Ouwens R. Fractures of the base of the first metacarpal bone: results of surgical treatment. Injury, 1989; 20: 359-62.

6. Brazier J, Moughabghab $M$, Migaud H. Les fractures articulaires de la base du premier métacarpien. Ann Chir Main, 1996; 15: 91-9.

7. Foster RJ, Hastings $\mathrm{H}$. Treatment of Bennett, Rolando, and vertical intraarticular trapezial fractures. Clin Orthop, 1987; 214: 121-9.

8. Langhoff $\mathrm{O}$, Andersen K, KjaerPetersen K. Rolando's fracture. J Hand Surg B, 1991; 16: 454-9.

9. Surzur P, Rigault M, Charissoux JL. Fractures récentes de la base du premier métacarpien. Ann Chir Main, 1994; 13: 12234.

10. Bruske J, Bednarski M, Niedzwiedz Z. The results of operative treatment of fractures of the thumb metacarpal base. Acta Orthop Belg, 2001; 67:368-73. 